\title{
A Study of Metallo-beta-lactamase Producing Pseudomonas aeruginosa Isolates in a Tertiary Care Hospital
}

\author{
Snehal Patil ${ }^{1}$, Anahita Hodiwala ${ }^{2 *}$ and Shailendra Patil ${ }^{1}$ \\ ${ }^{1}$ BKL Walawalkar Rural Medical College, Sawarde, Chiplun, Maharashtra, India \\ ${ }^{2}$ MGM Medical College, Maharashtra, India \\ *Corresponding author
}

\section{A B S T R A C T}

\section{Keywords}

Pseudomonas aeruginosa, Metallo-betalactmase, Carbapenem, Multidrug resistance

Article Info

Accepted:

07 March 2019

Available Online:

10 April 2019

\begin{abstract}
Metallo-beta-lactmase producing Pseudomonas aeruginosa strains are responsible for several nosocomial outbreaks in tertiary care centres across the world. It is well known that poor outcome occurs when patients with serious infections due to MBL producing organisms are treated with antibiotics to which the organism is completely resistant. Therefore, detection of MBL producing Pseudomonas aeruginosa strains is crucial for optimal treatment of critically ill patients and to prevent the spread of resistance. Aim of the present study is to detect Metallo-beta-lactmase (MBL) production in clinical isolates of Pseudomonas aeruginosa by Imipenem-EDTA Double Disc Synergy test. 100 strains of Pseudomonas aeruginosa isolated from pus, sputum, urinary catheter tip, blood and body fluids were screened for Carbapenem resistance by Kirby-Bauer disk diffusion method and results were interpreted as per CLSI guidelines. The isolates showing resistant to Imipenem were further tested for MBL production by Imipenem-EDTA Double Disc Synergy test. Out of 100 Pseudomonas aeruginosa strains 15 were resistant to Imipenem. Out of 15 isolates 10 were MBL producers. Proper antibiotic policy and measures to restrict the indiscriminative use of cephalosporins and carbapenems should be taken to minimize the emergence of Metallo-beta-lactmase producing pathogens.
\end{abstract}

\section{Introduction}

$P$. aeruginosa is a common nosocomial pathogen, notorious for its multidrug resistance (MDR) and life threatening infections in critically ill patients. It is aerobic Gram negative bacillus, highly versatile microorganism able to tolerate low oxygen conditions. It can survive with low levels of nutrients and grow in temperatures ranging from $4-42^{0} \mathrm{C}^{[1]}$. P. aeruginosa can cause pneumonia, urinary tract infections and bacteremia as well as causing high morbidity and mortality in patients with cystic fibrosis due to chronic infections that eventually cause pulmonary damage and respiratory insufficiency. Infections due to $P$. aeruginosa are difficult to eradicate because of their elevated intrinsic resistance as well as their capacity to acquire resistance to different antibiotics ${ }^{[2]}$

$P$. aeruginosa, a virulent microorganism is susceptible to only limited number of 
antibiotic agents. It accounts for about $11 \%$ of all nosocomial infections and ranks fifth among all the nosocomial pathogens. There are various mechanisms involved in the resistance of $P$. aeruginosa, among them over expression of efflux pump, acquisition of Extended-Spectrum beta Lactamases (ESBLs) and Metallo-beta-Lactamases; target site or outer membrane modification, por in mutations, plasmid enzymatic modifications $^{[3] \text {. }}$

MBL belongs to a group b-lactamase which requires divalent cations of zinc as cofactors for enzyme activity. IMP and VIM genes responsible for MBL production are transferable via plasmids and can rapidly spread to other bacteria. Over the last decade metallobeta lactamases (MBL) producing isolates have emerged particularly in Pseudomonas aeruginosa.

These isolates have been responsible for serious infections such as septicemia and pneumonia and have been associated with failure of therapy with carbapenems. In recent years MBL genes have spread from $P$. aeruginosa to Enterobacteriaceae, and a clinical scenario appears to be developing that could simulate the global spread of extendedspectrum beta-lactamases. Therefore, detection of MBL producing gram negative bacilli especially $P$. aeruginosa is crucial for optimal treatment of patient particularly critically ill and hospitalized patients and to control the spread of resistance.

The unique problem with MBLs is their unrivalled broad spectrum resistance profile. In addition in many cases the MBL gene may be located on plasmids with gene encoding other antibiotic resistance determinants. Hence the early detection of MBL producing $P$. aeruginosa may avoid the future spread of these multidrug resistant strains.

\section{Materials and Methods}

\section{Study design}

This study was conducted in the Department of Microbiology at MGM Medical College, Navi Mumbai, between January 2014 and January2015.

A total 100 clinical isolates were subjected to MBL detection method. Samples were collected from pus, sputum, urinary catheter tip, blood, pleural fluid, endotracheal secretions.

\section{Sample collection and processing}

Samples were collected in sterile, wide mouthed containers and then transferred to Microbiology Laboratory for further processing. Samples were cultured onto Pseudomonas isolation agar plates (Himedia).Colonies with an appropriate colonial morphologies were classified presumptively as $P$. aeruginosa and they were further identified by conventional biochemical tests. Antimicrobial susceptibility testing was done by Kirby Bauer disk diffusion method as per Clinical Laboratory Standard Institute (CLSI) guidelines. P. aeruginosa were stored in $1 \%$ nutrient agar slant at $4^{0}$ Centigrade for doing further analyses. The isolates showing resistant to Imipenem were further tested for MBL production by Imipenem-EDTA Double Disc Synergy test. The Imipenem-EDTA double disc synergy test was performed as described by Lee et al., ${ }^{[4]}$

\section{Imipenem-EDTA Double Disc Synergy Test (DDST)}

A $0.5 \mathrm{M}$ EDTA solution was prepared by dissolving $186.1 \mathrm{~g}$ of disodium EDTA.2H20 in $1000 \mathrm{ml}$ distilled water and adjusting it to $\mathrm{pH}$ 8.0 by using $\mathrm{NaOH}$. The mixture was sterilized by autoclaving. Direct colony 
suspension of test organism adjusted to match with 0.5 McFarland turbidity was prepared and inoculated into the Mueller-Hinton agar plate as recommended by National Committee for Clinical Laboratory Standards ${ }^{[5]}$ An Imipenem (10micro gram) disc was placed 20 $\mathrm{mm}$ centre to centre from a blank disc containing 10 microlitre of $0.5 \mathrm{M}$ EDTA (750 microgram).The inhibition zones of the Imipenem and EDTA disc were compared after 16-18 hours of incubation in air at $37^{\circ} \mathrm{C}$.Enhancement of zone of inhibition in the area between Imipenem and the EDTA disc in comparison with the zone of inhibition on the far side of the drug was interpreted as a positive result

\section{Results and Discussion}

Out of $100 P$. aeruginosa isolates 38 were from Pus followed by Sputum (23), Urine (12), ET Tip (6), Ear swab(6), Blood (4), Catheter tip (4), Pleural fluid (2), Bronchoalveolar lavage (2), ET Aspirate (1), Suction tip (1) and Tissue (1).

Out of 100 isolates 15 were resistant to Imipenem. Out of 15 Imipenem resistant isolates 4 were from Urine samples, followed by Pus (3), Sputum (2), ET tip (2), Blood (2), ET Aspirate (1) and Suction tip (1) (Fig. 1; Table 1).

Table.1 Total Imipenem resistant strains in clinical samples

\begin{tabular}{|c|c|c|}
\hline Sr No. & Samples & $\begin{array}{c}\text { Total Imipenem } \\
\text { resistant strains }\end{array}$ \\
\hline $\mathbf{1 .}$ & Pus & $\mathbf{3}$ \\
\hline $\mathbf{2 .}$ & Sputum & $\mathbf{2}$ \\
\hline $\mathbf{3 .}$ & Urine & $\mathbf{4}$ \\
\hline $\mathbf{4 .}$ & ET Tip & $\mathbf{2}$ \\
\hline $\mathbf{6 .}$ & Blood & $\mathbf{2}$ \\
\hline $\mathbf{1 0}$ & Endotracheal & $\mathbf{1}$ \\
\hline $\mathbf{1 1}$ & Aspirate & $\mathbf{1}$ \\
\hline & Suction Tip & $\mathbf{1 5}$ \\
\hline
\end{tabular}

Out of 100 P. aeruginosa 15 isolates were resistant to imipenem

Fig.1 Imipenem-EDTA DDST

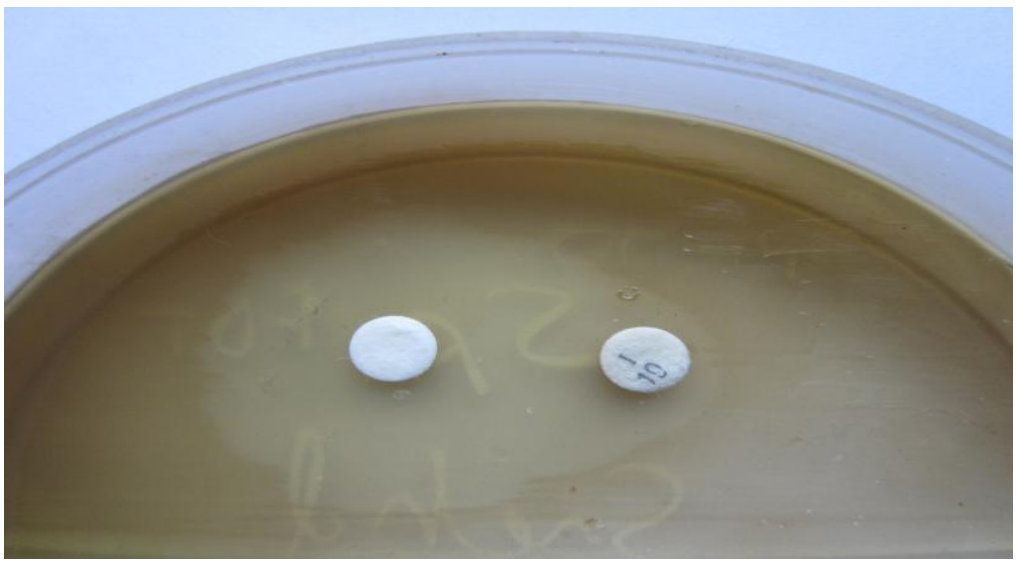




\section{Graph.1 Production of Metallo-beta-lactamase among clinical isolates of Pseudomonas} aeruginosa in clinical samples

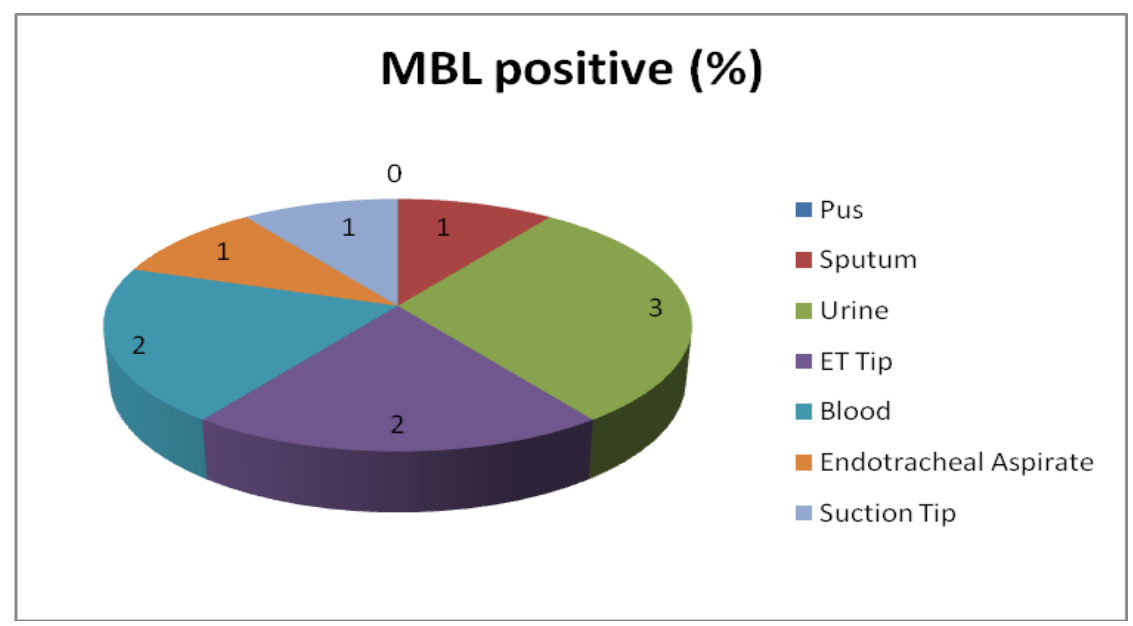

Out of 15 Imipenem resistant isolates 10 were found to be MBL producers by Imipenem EDTA DDST test. Among the $10 \mathrm{MBL}$ producing Pseudomonas aeruginosa isolates, maximum were from Urine (3), followed by ET Tip (2), Blood (2), Sputum (1), ET Aspirate (1) and Suction tip (1).

In our study, out of 100 isolates of $P$. aeruginosa, Amikacin (89\%) was found to be more sensitive, followed by Ciprofloxacin (85\%), Gentamycin (76\%), Ofloxacin (72\%), Lomefloxacin (70\%), Pefloxacin (62\%), Cefotaxime (62\%), Cefoperazone (57\%) and Ceftazidime (54\%), Imipenem (55\%).

Carbapenemases may be defined as betalatamases that significantly hydrolyze at least imipenem or meropenem. Carbapenam resistance due to production of metallo beta lactamases (MBL) in gram negative organisms is an increasing international public health problem. Over the last decade MBL producing isolates have emerged particularly in Pseudomonas aeruginosa. These isolates have been responsible for serious infections such as septicemia and pneumonia and have been associated with failure of therapy with carbapenems. $P$. aeruginosa producing MBL was first reported from Japan in $1991^{[7]} \mathrm{MBL}$ belongs to a group b-lactamase which requires divalent cations of zinc as cofactors for enzyme activity. IMP and VIM genes responsible for MBL production are transferable via plasmids and can rapidly spread to other bacteria ${ }^{[8]}$

Another study conducted by Shashikala et al., [9] reported $20.7 \%$ carbapenem resistant Pseudomonas aeruginosa isolates from endotracheal aspirates showing indwelling devices as major risk factors for the development of resistance.

Supriya Upadhyay et al., ${ }^{[10]}$ studied different beta-lactamase classes among clinical isolates of Pseudomonas aeruginosa expressing AmpC beta-lactamase enzyme and reported 120 (59.4\%) isolates were positive for AmpC betalactamase. Among them, 14 strains (7\%) were inducible AmpC producers. Co-production of AmpC along with extended spectrum betalactamase and metallo beta-lactamase was reported in $3.3 \%$ and $46.6 \%$ isolates respectively.

In our study 15 out of $100 P$. aeruginosa isolates were resistant to imipenem. 10 out of 15 were found to be MBL producers by Imipenem EDTA DDST test. Five out of 15 were found to be MBL non producers by the Imipenem EDTA DDST test. 
Our findings are similar with study done by Deeba Bashir et al., ${ }^{[11]}$ It was observed by them that Out of $283 P$. aeruginosa isolates, 38 $(13.42 \%)$ were resistant to Imipenem. Thirty three $(11.66 \%)$ were found to be MBL producers by combined disk test and all of them showed reduction in MIC in the presence of imipenem-EDTA in Etest.

Similarly, In 2002 Navneeth et al., ${ }^{[12]}$ from India first reported MBL production in $P$. aeruginosa to be 12 per cent.

However our results are not in agreement with Ami Varaiya et al., ${ }^{[13]}$ who reported incidence of metallo beta lactamase producing Pseudomonas aeruginosa in ICU patients. They found that out of the $240 P$. aeruginosaisolates60 (25\%) were found to be carbapenem resistant and 50(20.8\%) were found to be MBL producers.

In the present study, Among the 10 of MBL producing Pseudomonas aeruginosa, maximum strains were from urine, followed by ET Tip, Blood, Sputum, ET Aspirate and Suction tip. MBL positive isolates were recovered from Urine. Most of these patients were having indwelling urinary catheter.

In the present study, antimicrobial resistance pattern of the Pseudomonas aeruginosa isolates showed maximum resistance against Meropenem (80\%), followed by Cefuroxime (77\%), Cefepime (75\%), Ticarcillin (70\%), Ceftriaxone + Tazobactum $(65 \%)$ and Cefoperazone + Sulbactum (65\%), Imipenem $(45 \%)$.

In this study, Pseudomonas aeruginosa showed higher sensitivity to Amikacin (89\%), Ciprofloxacin (85\%), Gentamycin $(76 \%)$, Ofloxacin (72\%), Lomefloxacin (70\%), Pefloxacin (62\%), Cefotaxime (62\%), Cefoperazone $(57 \%)$ and Ceftazidime $(54 \%)$, Imipenem (55\%). Similarly Javiya et al., demonstrated maximum sensitivity to amikacin against Pseudomonas species ${ }^{[14]}$
In the present study results of antibiotic susceptibility test showed that multidrug resistant ability of $P$. aeruginosa. Also Carbapenem resistance not only has enormous therapeutic implications, but is also important from the point of view of infection control. Such strains are known for rapid intra institutional spread and therefore, must be notified to infection control team. The present study was conducted with above perspective in view to know the prevalence of MBL producing $P$. aeruginosa and possible treatment alternatives. As more and more MBL-producing Pseudomonas aeruginosa isolates are being reported as an important cause of nosocomial infections, appearance of MBL genes and their spread among bacterial pathogens is a matter of concern with regard to the future of antimicrobial therapy.

\section{References}

1. Stover C.K., Pham X.Q., Erwin A.L., Mizoguchi S.D., Warrener P., Hickey M.J., et al., 2000. Complete genome sequence of Pseudomonas aeruginosa PAO1, an opportunistic pathogen. Nature, 406: 959-964.

2. Breidenste, in EBM, De la Fuente-Nunez C, Hancock REW 2011. Pseudomonas aeruginosa: all roads lead to resistance. Trends Microbiol, 19:419-426.

3. Manu Chaudhary, Anurag Payasi. Rising Antimicrobial Resistance of Pseudomonas aeruginosa Isolated from Clinical Specimens in India. $\mathbf{J}$ Proteomics Bioinform 6: 005-009.

4. Lee K, Lim Y.S., Yong D, Yum Jh, Chong Y 2003.Evalution of the Hodge test and the imipenem-EDTA double disc synergy test for differentiation of metallo-beta-lactamases producing clinical isolates of Pseudomonas spp. and Acinetobacter spp. J Clin Microbio, 41: 4623-9.

5. Wayne, Pa. National committee for clinical laboratory standards. Performance standards for antimicrobial susceptibility testing. Eleventh 
informational supplement. 2001

6. Cornalgia G., Akova, M., Amicosante G 2007. Study Group for Antimicrobial Resistance Surveillance. Metallo-betalactamases as emerging resistance determinants in Gram negative pathogens. Int $\mathbf{J}$ Antimicrob Agents, 29:380-8.

7.Yano, H, Kuga A, Okamota R, Kitasato H, Kobayashi T, InonM2001. Plasmid coded metallo beta lactamase (imp 6) conferring resistance to carbapenems, especially meropenam. Antimicrob Agents Chemother, 4: 1343-8.

8. Bush, K., 1998. Metallo b-lactamase: a class apart. Cli Infect Dis, 27(1): 48-53.

9. Shashikala, Kanungo R, Srinivasan S, Devi S 2006. Emerging resistance to carbapenem in hospital acquired Pseudomonas infection; A cause of concern; Indian J. Pharmacol,38: 287 8.

10. Supriya Upadhyay, Malay Ranjan Sen, Amitabha Bhattacharjee 2010. Presence of different beta-lactamase classes among clinical isolates of Pseudomonas aeruginosa expressing AmpC betalactamase enzyme. J Infect Dev Ctries, 4(4): 239-242.

11. Deeba Bashir, Manzoor Ahmad Thokar. Detection of metallo-beta-lactamase (MBL) producing Pseudomonas aeruginosa at a tertiary care hospital in Kashmir. African Journal of Microbiology Research Vol. 5(2): 164172.

12. Navneeth, BV, Sridaran D, Sahay D, Belwadi MR 2002. A preliminary study on metallobeta lactamse producing Pseudomonas aeruginosa in hospitalized patients. Indian J Med Res,
116: 264-7.

13. Ami Varaiya, Nikhil Kulkarni, Manasi Kulkarni, Pallavi Bhalekar, Jyotsana Dogra 2008. Incidence of metallo beta lactamase producing Pseudomonas aeruginosa in ICU patients. Indian J Med Res, 398-402.

14. Javiya, V.A., Ghatak S.B., Patel K.R., Patel JA 2008. Antibiotic susceptibility patterns of Pseudomonas aeruginosa at a tertiary care hospital in Gujarat, India. Indian J. Pharmacol, 40: 230-4.

15. Mackie, T.J., McCartney JE 2008. Laboratory Strategy in the diagnosis of infective syndrome. In: Mackie \& McCartney Practical Medical Microbiology.14th edition. Collee JG, Froser AG, Marmian BD, Simmons A, editors. New Delhi, India : Churchill Livingstone,53-94.

16. Betty A. Forbes, Daniel, F Sahm2007.Overview of bacterial identification methods and strategies. In Bailey \& Scott's Diagnostic Microbiology. 12th ed. Lorren Wilson, Ellen Wurm, editors. St. Louis, Missouri USA: Mosby Elsevier; 218-47.

17. Wayne, Pa., National committee for clinical laboratory standards. Performance standards for antimicrobial susceptibility testing. Eleventh informational supplement. 2001

18. Simpson, JA, Smith SE, Dean RT 1989. Scavenging by alginate of free radicals released by macrophages. Free Radic Biol Med, 6: 347-53.

19. Leigh, R, Evans, Alfred Linker 1973.Production and characterization of the slime polysaccharide of the Pseudomonas aeruginosa. J Bacteriol, 116:915-24.

\section{How to cite this article:}

Snehal Patil, Anahita Hodiwala and Shailendra Patil. 2019. A Study of Metallo-beta-lactamase Producing Pseudomonas aeruginosa Isolates in a Tertiary Care Hospital. Int.J.Curr.Microbiol.App.Sci. 8(04): 600-605. doi: https://doi.org/10.20546/ijcmas.2019.804.065 